\title{
Ethical considerations in the management of Ebola virus disease
}

T he current Ebola virus disease (EVD) outbreak in West Africa is the largest since the disease was first described in 1976. By the end of March this year, it had caused more than 10000 deaths, including 495 among health care workers (HCWs). ${ }^{1,2}$ Twenty-four patients with EVD have been admitted to hospitals in high-income countries. ${ }^{3}$ The nurses who were infected while looking after Thomas Duncan in Dallas, Texas, highlighted the risk of transmission of EVD to HCWs, even in well resourced hospitals. HCWs in Australia were quick to compare this with an outbreak of severe acute respiratory syndrome (SARS) in a Toronto hospital in 2003, when a single case led to an epidemic in which nearly half the cases occurred in HCWs. ${ }^{4}$

\section{"it is crucial that the decision-making process is transparent, ethically and clinically rigorous, and acceptable to all stakeholders"}

The public response after a doctor who became infected while treating patients with EVD in Africa returned to the United States, and the ostracising of HCWs at the hospital where he was treated, reflected community concerns about EVD. ${ }^{5}$ The unpredictable nature of EVD, the infection of carers and the high case-fatality rate in Africa have generated fear of the disease.

HCWs have raised concerns about appropriate clinical management of patients with EVD and the need for a clear ethical framework to guide decision making. ${ }^{6}$ While there are general management guidelines for patients with EVD, including strict isolation, appropriate use of personal protective equipment (PPE) and prompt correction of fluid and electrolyte loss, uncertainty surrounds many aspects of care, including the extent to which supportive care can be provided and the circumstances under which it should be discontinued.

Here, we focus on two ethical questions regarding the critical care of patients with EVD in an Australian setting. The first is whether it is ethically appropriate in some circumstances for HCWs to decline to care for patients with EVD. The second question concerns how treatment decisions should be made regarding limitation of therapy for patients with EVD. Similar questions have been asked in the context of other infectious diseases, including SARS, where provision of care put HCWs at significant risk, and in the early stages of the AIDS epidemic, when the risk to HCWs was minimal. ${ }^{7,8}$
Summary

The large-scale outbreak of Ebola virus disease (EVD) in West Africa, with subsequent cases in high-income countries, has focused international attention on this disease.

Health care workers (HCWs) have raised concerns about appropriate clinical management of patients with EVD and the need for a clear ethical framework to guide decision making.

There are two main ethical questions regarding the critical care of patients with EVD in an Australian setting:

- Is it ethically appropriate in some circumstances for HCWs to decline to care for patients with EVD?

- How should treatment decisions be made regarding limitation of therapy for patients with EVD?

The key concern is ensuring that no patient is denied therapy that should be provided, while preventing unnecessary risk to HCWs.

It is imperative to develop an approach that facilitates rigorous, evidence-based and ethically justifiable decision making, which should include a predetermined, institutionally endorsed process for assessing difficult clinical scenarios as they arise.

Is it ever ethically appropriate to decline to care for patients with Ebola virus disease?

Questions regarding the critical care of patients with EVD will not be resolved simply by recourse to the concept of futility. ${ }^{9}$ The frequently quoted EVD case-fatality rate of $60 \%-90 \%$ now appears excessive, as Médecins sans Frontières have reported case-fatality rates in Guinea of $25 \%$ (among 21 patients treated in Telimele) and 37\% (among 59 confirmed cases in Donka)..$^{10}$ Of the 24 patients managed in Western countries so far, five $(21 \%)$ have died, all of whom were seriously ill on admission to hospital. ${ }^{3}$ These data favour an aggressive approach to treatment.

However, the lack of definitive therapy and the devastating effects of infection are sobering realities that counterbalance the argument for aggressive therapy and complicate the dilemma faced by clinicians. Emergency department, infectious diseases, laboratory and intensive care personnel are exposed to higher risk than other HCWs. Nurses, in particular, spend long periods in contact with patients and are therefore most vulnerable. Although the risks to HCWs cannot be easily quantified, they can be minimised to acceptable levels with protocols, training, 
optimal staffing ratios, appropriate use of PPE and integrated teamwork. The key question for HCWs and policymakers becomes one of defining what constitutes an acceptable level of risk, and what obligations HCWs have to care for patients with high-risk illnesses. ${ }^{11}$

Health professionals have codes of conduct that identify the concept of duty of care but provide no guidance as to how it applies in situations involving significant personal risk. ${ }^{12} \mathrm{HCWs}$ must personally decide how to balance their duty to a highly infectious patient against their duty to protect themselves. While such decisions are often seen as binary - HCWs have either a "duty to treat" or a "right to refuse" - in fact, they are highly complex. These decisions are subject to multiple influences, including how clinicians perceive the risk of exposure to EVD and how well they tolerate risk in general; how they prioritise their own family responsibilities; how well they trust their colleagues, the hospital as an organisation and the PPE provided; and how they perceive their professional, ethical and legal responsibilities to their patients and employer. ${ }^{13-15}$

Data on willingness to treat patients with high-risk illnesses show interesting patterns. Among 1000 physicians surveyed in the US, $80 \%$ indicated willingness to care for patients with an unknown but lethal illness, but only about $20 \%$ felt prepared to do so. ${ }^{15}$ Psychometric data in risk perception demonstrate that what appears to be a neutral, unbiased and professional risk assessment will be affected by cognitive heuristics, which can produce systematic distortions in thinking. ${ }^{16}$ Scientific experts also display such thinking, and retrospective assessments of responses to crisis events routinely identify "over-reactions".

Ethical considerations relating to providing supportive therapy for patients with EVD appear straightforward. There is an obligation to treat patients with therapies that provide the greatest likely benefit, with manageable risks to staff and the institution. However, this expectation is not unlimited: it also depends on whether adequate resources are provided to allow staff to work in the safest possible environment. Employers have a responsibility to provide these resources and could not ethically compel staff to care for patients if doing so exposed them to avoidable personal risk. ${ }^{17}$ However, whether they could do so in these circumstances would depend on how "adequate resources" and "avoidable risk" were defined.

While some staff might prefer not to care for a patient with EVD because of a perceived high level of personal risk, experience in the current EVD outbreak and previous epidemics, notably SARS, suggests that HCWs rarely refuse if provided with adequate information, training, resources and rosters. Nevertheless, a small number of HCWs may need to be excused from caring for patients with EVD because of underlying medical or psychological factors.
How should treatment decisions for patients with Ebola virus disease be made?

As therapies for a seriously ill patient become progressively more invasive, the relative benefits diminish while the risk to carers increases. For patients with EVD, aerosol-generating procedures, such as mechanical ventilation, place HCWs at increased risk. However, compared with non-invasive ventilation, placement of an endotracheal tube and closed-circuit ventilation decrease the risk, particularly in patients with haemoptysis.

In other circumstances, the benefit to the patient of aggressive supportive care may be more difficult to determine. Dialysis, for example, poses several challenges. Independent of the prognosis of acute renal failure in the setting of severe EVD, ${ }^{18}$ it would seem unethical not to offer dialysis. While treatment protocols for renal replacement therapy have been developed, ${ }^{19}$ the risks of individual therapies have not been quantified. However, patients with EVD have been treated experimentally with plasmapheresis and continuous renal replacement therapy, without any reports of cross-infection. ${ }^{20}$

While the clinical obligations of HCWs are aligned to maximise therapeutic options for patients, this is not necessarily the case for other aspects of care. Despite detailed protocols for risk assessment and safe collection, transport and laboratory testing of specimens from patients with EVD, laboratory investigations and blood transfusion are often limited to point-of-care modalities and uncross-matched donor supplies, respectively. The relevant protocols involve strict precautions, comparable with those for clinical management of patients with EVD. ${ }^{21}$ Although transmission of EVD in laboratory settings has only been documented when breaches of protocol have occurred, ${ }^{22-24}$ laboratory directors may be unwilling to subject staff to any perceived risk or to incur potential disruption to the rapid throughput of routine specimens. However, protocols that restrict laboratory testing limit investigative and therapeutic options available to clinicians and potentially compromise patient outcomes. We contend that the ethical obligations of frontline clinical staff to provide patient care should also apply to laboratory staff.

Imaging for patients with EVD is also restricted, usually to bedside radiography or ultrasonography, and it is unlikely that a patient would be considered for a major surgical intervention or cross-sectional imaging under current institutional protocols. These protocols require that, once admitted, a patient with EVD should remain in the ultra-isolation unit until fully recovered. This requirement is based on the potential risk, however small, to staff, patients or the public from exposure to a patient with EVD being transported within the hospital.

Invasive or aggressive interventions would be contraindicated, as they would be for any patient, if 
continued care of a patient with EVD were judged to be futile. However, the potential increased risk to HCWs would add weight to an otherwise marginal decision to cease active intervention. Compassionate palliation would then be indicated. For example, a patient with EVD who has a massive haemorrhage could pose an enormous risk to HCWs. Likewise, cardiopulmonary resuscitation, which may be performed as a last resort for patients with other illnesses, would be inappropriate for patients with EVD. Recent ethical debate in the US about the role of cardiopulmonary resuscitation in patients with EVD canvassed the problem of inevitable delay because of the requirement for resuscitation teams to don PPE, but cautioned that an automatic "do not resuscitate" order may not be appropriate in the US. ${ }^{25}$

\section{How can clinicians resolve these dilemmas?}

Ethical debate during the SARS epidemic resulted in a broad consensus that HCWs have a duty to treat patients with infectious diseases, but that this duty is not absolute and is dependent on the degree of risk to which HCWs are exposed, their capacity to protect themselves from the risk, their skills and expertise, and the specific circumstances. ${ }^{11}$

Situations may arise where a decision not to treat or not to escalate therapy is justifiable because of unmanageable risk, despite all available resources. In such situations, where it is difficult to extricate the ethical obligations owed to patients from those owed to staff, and decisions may be contestable, it is crucial that the decision-making process is transparent, ethically and clinically rigorous, and acceptable to all stakeholders. These conditions are likely to be met if decisions are not made by a single clinician at the bedside but are based on a clear institutional policy or advice from an expert advisory group.

For clinicians, the answers to many of these ethical questions will be clarified as experience in the care of patients with EVD in Western hospitals grows. Just as it did in the setting of HIV and SARS, the hysteria associated with EVD is likely to settle over time and as effective therapeutic options become available. In the interim, it is imperative to develop an approach that facilitates rigorous, evidence-based and ethically justifiable decision making. In this way, we can optimise clinical care, maintain the integrity and efficient functioning of the health system, and reduce stigma and professional and public anxiety.

While issues relating to other infectious diseases, such as extremely drug-resistant tuberculosis and virulent respiratory viral infections, have been debated, ${ }^{26}$ particularly difficult questions arise in relation to the critical care of patients with EVD, due to its severity, the absence of proven therapies and the risk to HCWs. The key concern is ensuring that no patient is denied therapy that should be provided, while preventing unnecessary risk to staff. It is possible that these may represent intersecting priorities, and the balance would, of necessity, favour preventing infections in staff.

These dilemmas can be resolved with an approach that includes a predetermined, institutionally endorsed process for assessing difficult clinical scenarios as they arise. This process should be transparent and consistent, with clear policies and governance structures to provide the optimal balance between ensuring a patient with EVD receives appropriate care and that staff are not placed at unnecessary risk.

Competing interests: No relevant disclosures.

Provenance: Not commissioned; externally peer reviewed.

(c) 2015 AMPCo Pty Ltd. Produced with Elsevier B.V. All rights reserved.

References are available online at www.mja.com.au. 
1 World Health Organization. Ebola situation report - 1 Apr 2015. http://apps. who.int/ebola/current-situation/ebola-situation-report-l-april-2015-0 (accessed Apr 2015).

2 Parra JM, Salmerón OJ, Velasco M. The first case of Ebola virus disease acquired outside Africa. N Engl J Med 2014; 371: 2439-2440.

3 Ashkenas J, Buchanan L, Burgess J, et al. How many Ebola patients have been treated outside of Africa? New York Times 2015; 26 Jan. http://www. nytimes.com/interactive/2014/07/31/world/africa/ebola-virus-outbreakqa.html (accessed Jul 2015).

4 SARS Commission. Final report. Volume 2 - spring of fear. Chapter 3: the story of SARS. Vancouver: a tale of two cities. Toronto: Publications Ontario, 2003: 245-297. http://www.archives.gov.on.ca/en/e_records/sars/ report/v2.html (accessed Apr 2015).

5 Hartocollis A, Schweber N. Bellevue employees face Ebola at work, and stigma of it everywhere. New York Times 2014; 29 Oct. http://www. nytimes.com/2014/10/30/nyregion/bellevue-workers-worn-out-fromtreating-ebola-patient-face-stigma-outside-hospital.html (accessed Apr 2015).

6 Rubin EJ, Baden LR. Out of Africa - caring for patients with Ebola. NEngl J Med 2014; 371: 2430-2432

7 Dwyer J, Tsai DF. Developing the duty to treat: HIV, SARS, and the next epidemic. J Med Ethics 2008; 34: 7-10.

8 Minkoff H. Ecker J. Physicians' obligations to patients infected with Ebola: echoes of acquired immune deficiency syndrome. Am J Obstet Gynecol 2015; 212: 456.el-4. doi: 10.1016/j.ajog.2014.12.026.

9 Schneiderman LJ. Defining medical futility and improving medical care. J Bioeth Inq 2011; 8: 123-131. doi: 10.1007/s11673-011-9293-3.

10 Médecins Sans Frontières. Our work. Medical issues: Ebola. http://www. doctorswithoutborders.org/our-work/medical-issues/ebola (accessed Feb 2015).

11 Reid L. Diminishing returns? Risk and the duty to care in the SARS epidemic. In: Selgelid MJ, Battin MP, Smith CB, editors. Ethics and infectious disease. Malden, Mass: Blackwell, 2006: 171-183.

12 Kerridge I, Lowe M, Stewart C. Ethics and law for the health professions. 4th ed. Sydney: Federation Press, 2013.

13 Taversky A, Kahneman D. Judgement under uncertainty: heuristics and biases. Science 1974; 185: 1124-1131.
14 Sokol DK. Virulent epidemics and scope of healthcare workers' duty of care. Emerg Infect Dis 2006; 12: 1238-1241.

15 Alexander GC, Wynia MK. Ready and willing? Physicians' sense of preparedness for bioterrorism. Health Aff (Millwood) 2003; 22: 189-197.

16 Kahneman D, Tversky A. Prospect theory: an analysis of decisions under risk. Econometrica 1979; 47: 263-292. doi: 10.2307/1914185.

17 Ruderman C, Tracy CS, Bensimon CM, et al. On pandemics and the duty to care: whose duty? who cares? BMC Med Ethics 2006; 7: 5. doi: 10.1186/14726939-7-5.

18 Gallagher M, Cass A, Bellomo R, et al; POST-RENAL Study Investigators and the ANZICS Clinical Trials Group. Long-term survival and dialysis dependency following acute kidney injury in intensive care: extended follow-up of a randomized controlled trial. PLOS Med 2014; 11: e1001601.

19 Connor MJ Jr, Kraft C, Mehta AK, et al. Successful delivery of RRT in Ebola virus disease. J Am Soc Nephrol 2015; 26: 31-37. doi: 10.1681/ASN.2014111057.

20 Clark DV, Jahrling PB, Lawler JV. Clinical management of filovirus-infected patients. Viruses 2012; 4: 1668-1686.

21 Public Health Laboratory Network. Laboratory procedures and precautions for samples collected from patients with suspected viral haemorrhagic fevers. Canberra: Australian Government Department of Health. Nov 2014. http://health.gov.au/internet/main/publishing.nsf/Content/cda-pubsother-vhf.htm (accessed Feb 2015).

22 Formenty P, Hatz C, Le Guenno B, et al. Human infection due to Ebola virus, subtype Côte d'Ivoire: clinical and biologic presentation. J Infect Dis 1999; 179 Suppl 1: S48-S53. doi: 10.1086/514285.

23 Miller J. Russian scientist dies in Ebola accident at former weapons lab. New York Times 2004; 25 May. http://www.nytimes.com/2004/05/25/world/ russian-scientist-dies-in-ebola-accident-at-former-weapons-lab.html (accessed Jul 2015).

24 Katz LM, Tobian AA. Ebola virus disease, transmission risk to laboratory personnel, and pretransfusion testing. Transfusion 2014; 54: 3247-3251.

25 Fins JJ. Responding to Ebola: questions about resuscitation. Bioethics Forum 2014; 10 Oct. http://www.thehastingscenter.org/Bioethicsforum/ Post.aspx?id=7135\&blogid=140 (accessed Jan 2015).

26 World Health Organization. Ethical issues in tuberculosis prevention, care and control. Oct 2014. http://www.who.int/tb/publications/ethics_in_tb factsheet_28janllrev.pdf (accessed Jul 2015). 